\title{
(2) OPEN ACCESS \\ Successful rescue treatment of right posterior inferior cerebellar artery (PICA) vascular stasis with tirofiban following endovascular coil implantation
}

\author{
Christopher Houle $\odot$,' Varun Reddy ${ }^{2}$
}

${ }^{1}$ Critical/Neurocritical Care, Mohawk Valley Health System, Utica, New York, USA

${ }^{2}$ Neurointerventional Surgery and Comprehensive Stroke, Mohawk Valley Health System, Utica, New York, USA

Correspondence to Dr Christopher Houle; choule1@mvhealthsystem.org

Accepted 18 November 2020

\section{SUMMARY}

This report describes a patient who developed intraprocedural vascular stasis immediately following elective endovascular coil emboliation. Urgent antiplatelet treatment with the Gpllb/llla agent tirofiban was used. It was infused intra-arterially during the procedure, followed by a fixed rate intravenous continuous infusion, and successfully restored normal circulation. There were no reports of further bleeding or haemodynamic compromise during the hospital stay. The patient's condition returned to baseline and he was discharged the following day with no neurological deficits.

\section{BACKGROUND}

Thromboembolic events are common during endovascular coil embolisation and can increase mortality in neuroendovascular procedures. ${ }^{1}$ No standard dosing regimen currently exists for consecutive intra-arterial and intravenous tirofiban during coil embolisation rescue therapy.

\section{CASE PRESENTATION}

A man in his 60s presented for elective endovascular repair of a right posterior inferior cerebellar artery (PICA) aneurysm known to be causing thromboembolic phenomena (figure 1). A transradial approach was employed for catheterisation and endovascular repair of the aneurysm.

A $300 \mathrm{mg}$ clopidogrel and $325 \mathrm{mg}$ aspirin loading dose was administered prior to the procedure. On placing the patient under general anaesthesia in the angiography suite, placement of the microcatheter and primary embolisation of the aneurysm was successfully performed using Stryker target coils without incident. A total of 4200 units of heparin, in the form of heparinised saline, was administered during the procedure. Subsequently, a follow-up angiogram via the vertebral artery-guided catheter demonstrated occlusion of blood flow in the right PICA from the region of the newly implanted coils (figure 2).

Rescue therapy was promptly initiated using repeated doses of $0.1 \mathrm{mg}$ tirofiban, administered intra-arterially. A total of $0.3 \mathrm{mg}$ of tirofiban, was administered over $20 \mathrm{~min}$ directly into the PICA region. A follow-up angiogram was obtained showing improved perfusion and the microcatheter was removed without incident (figure 3).

Subsequent to the procedure, a tirofiban infusion was started at the fixed rate of $0.1 \mu \mathrm{g} / \mathrm{kg} / \mathrm{min}$ when

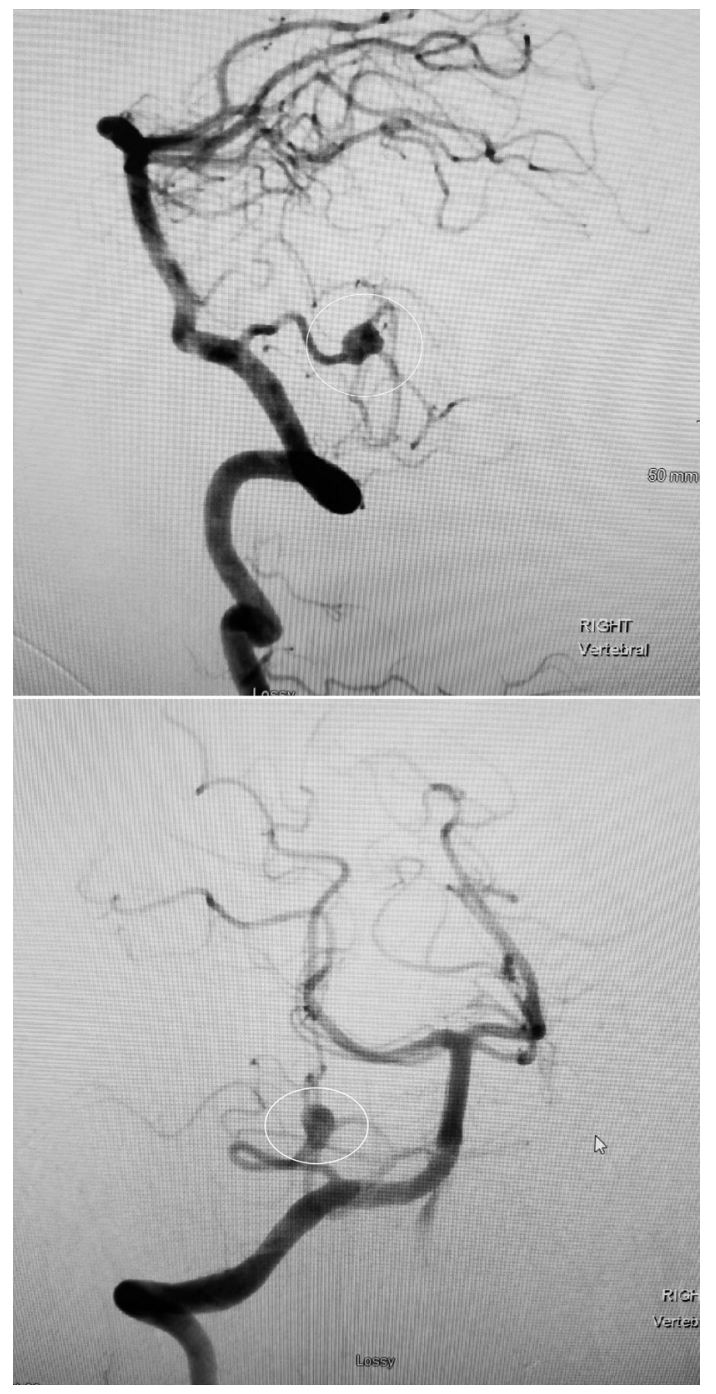

Figure 1 Initial working projection angiography showing aneurysm and the right posterior inferior cerebellar artery (lateral and anteroposterior views).

the measured anti-Xa level returned to therapeutic range. The infusion was continued for 15 hours. No adverse drug events were observed.

\section{OUTCOME AND FOLLOW-UP}

The patient presented for follow-up 30 days after primary coiling. He was alert and oriented, with no loss of function, in no acute distress and stated that he is quite active physically. (National Institutes of Health score $=0$, mRankin $=0$ ). He remains on the aspirin and clopidogrel combination. He noted 


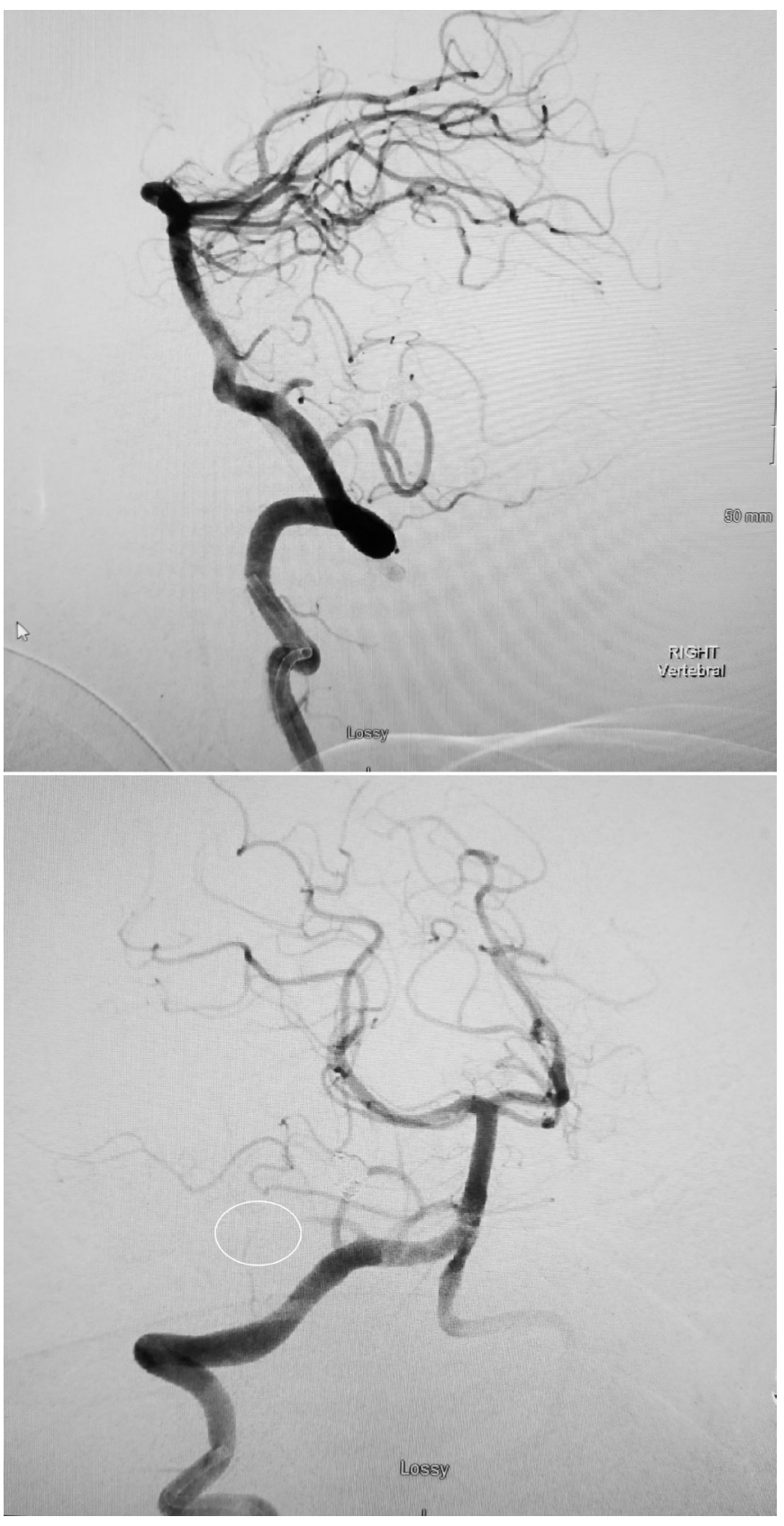

Figure 2 Post coiling. There is occlusion of the lateral medullary segments of the right posterior inferior cerebellar artery (lateral and anteroposterior views).

no significant side effects, except for some initial dizziness on standing during the immediate postoperative phase.

\section{DISCUSSION}

Thromboembolic events remain one of the most significant adverse events during coil embolisation for intracerebral aneurysm and range in frequency from $2 \%$ to $15 \% .^{2}$ GpIIb/IIIa agents, specifically eptifibatide and tirofiban, have been reported to be effective in eradication of periprocedural thrombus. ${ }^{13}$

Tirofiban is a short-acting, reversible glycoprotein (GpIIb/ IIIa) platelet function inhibitor that has been studied extensively for acute coronary syndrome (ACS), with a half-life of approximately 2 hours. Confirmation of efficacy and safety of tirofiban in ACS has been long established. From a standpoint of safety during neuroendovascular procedures, the short half-life and

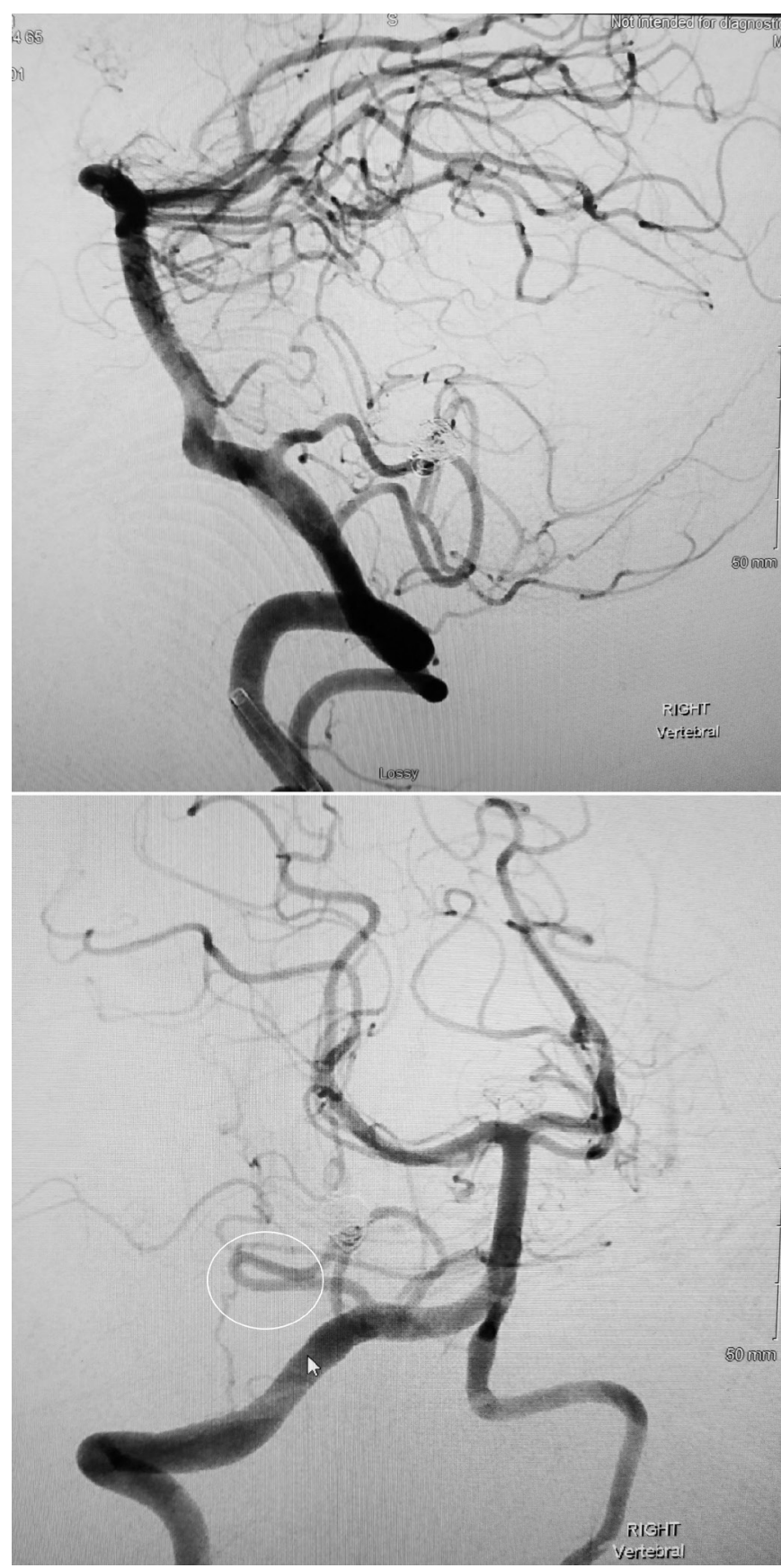

Figure 3 Angiography resolved. Successful recanalisation of lateral medullary segment of the right posterior inferior cerebellar artery (lateral and anteroposterior views).

reversible binding attributes, are an advantage with respect to abciximab, which has a longer half-life and irreversible binding. These attributes, combined with the low detrimental effect on postoperative bleeding, make it an attractive candidate for rescue therapy during coil embolisation. ${ }^{45}$

GpIIb/IIIa inhibitors have been used to treat coil embolisation thrombus both intravenously and intra-arterially using various methods and dosing combinations. ${ }^{6}$ An intravenous loading infusion followed by pulsatile intra-arterial injections, or typically intra-arterial injections alone, have been described. ${ }^{7}$ Additionally, there is at least one report of simultaneous intravenous and intra-arterial loading administration of tirofiban followed by maintenance therapy. ${ }^{1}$ Intra-arterial administration appears 
to be preferred over intravenous, due to intra-arterial access being most proximal to the thrombus and the risk of dosedependent complications, such as a cerebral haemorrhage, may be reduced. ${ }^{8}$ To our knowledge, there is currently no ideal standard dosing regimen that has been established for using GpIIb/ IIIa inhibitors to treat acute thromboembolism during endovascular procedures.

In this instance, the flow disturbance was presumably caused by an irregular coil surface or coil protrusion into the vessel lumen, resulting in vessel narrowing and subsequent thromboembolism via platelet aggregation. This happened in spite of the fact that the patient was loaded with a P2Y 12 inhibitor and aspirin just prior to the procedure and received intraprocedural heparin per protocol. It is worth noting that we do not currently have access to point-of-care (POC) testing for platelet inhibition at our institution. POC testing methods, such as VerifyNow (Accumetrics), can be used to measure platelet function and screen for responsiveness to help determine the optimal dosing and duration of antiplatelet agents prior to procedure.

During the procedure, the activated clotting time (ACT) was measured as high as $336 \mathrm{~s}$. When the thrombus was identified, intra-arterial tirofiban was given in $0.1 \mathrm{mg}$ aliquots until successful recanalisation. Due to the risk of lingering thrombus at the aneurysm site, it was decided that the patient was a candidate for post-treatment with a continuous infusion of tirofiban post procedure. However, the combination of GpIIb/IIIa inhibitors can prolong ACT, and the combination of heparin with a GpIIb/IIIa can increase the likelihood of both major and minor bleeding when compared with heparin alone. ${ }^{9}{ }^{10}$ Data from the primary Percutaneous Coronary Intervention (PCI) literature indicate a $49 \%$ increase in minor bleeding and a trend towards a $22 \%$ increase in major bleeding when GpIIb/IIIa inhibitors are combined with heparin. ${ }^{10}$ Due to these bleeding risks, and the absolute need to avoid cerebral haemorrhage post endovascular repair, the decision was made to delay the intravenous tirofiban infusion post procedure until the measured heparin activity was within normal limits. We chose to measure the anti-Xa level over

\section{Learning points}

- Fixed dosing of tirofiban was shown to be safe and efficacious in resolving thrombosis during this coil embolisation procedure.

- The combination of intravenous and intra-arterial tirofiban was successfully used without any complications, or adverse effects, in a patient who received loading doses of clopidogrel and aspirin, who also was heparinised.

- For those patients who are at high risk of thrombus post procedure, the continuous infusion of tirofiban can be initiated post embolisation during recovery in the intensive care unit.

- Newer point-of-care testing methods may help to identify poor responders to P2Y 12 and aspirin therapy, allow for dosing to be individualised prior to endovascular procedures, and reduce the risk of intraprocedural complications. several hours, and once it returned to a baseline level (0.1 IU/ $\mathrm{mL}$ ), the tirofiban infusion was initiated at $0.1 \mu \mathrm{g} / \mathrm{kg} / \mathrm{min}$ and continued for 15 hours post surgery. No thrombocytopenia was noted; platelet count was measured at $169 \times 10^{3} \mu \mathrm{l}$ at the end of the infusion.

Intra-arterial bolus dosing allows for focal drug delivery at the site of the thrombus. The exact method, and dosing scheme for rescue therapy, appears to vary depending on the clinician's experience and preference. In this case, giving tirofiban as an intra-arterial bolus + intravenous infusion protocol produced a positive outcome by allowing for thrombus obliteration and complete recanalisation, without unwanted bleeding or thrombocytopenia.

Acknowledgements The authors would like to express their appreciation to $\mathrm{Dr}$ Vikas Patel for his expert assistance and advice.

Contributors Conception or design of the work: VR and CH. Data collection: $\mathrm{CH}$. Data analysis and interpretation: VR and $\mathrm{CH}$. Drafting the article: $\mathrm{CH}$ and VR. Contributing editors: VR and CH. Final approval of the version to be published: VR.

Funding Medicure (grant number: 325).

Competing interests $\mathrm{CH}$ reports a grant from Medicure. VR reports personal fees from Janssen Pharmaceuticals.

\section{Patient consent for publication Obtained.}

Provenance and peer review Not commissioned; externally peer-reviewed.

Open access This is an open access article distributed in accordance with the Creative Commons Attribution Non Commercial (CC BY-NC 4.0) license, which permits others to distribute, remix, adapt, build upon this work non-commercially, and license their derivative works on different terms, provided the original work is properly cited and the use is non-commercial. See: http://creativecommons.org/ licenses/by-nc/4.0/

\section{ORCID iD}

Christopher Houle http://orcid.org/0000-0003-4185-745X

\section{REFERENCES}

1 Kim SH, Kim TG, Kong MH. Intra-Arterial and intravenous tirofiban infusion for thromboembolism during endovascular coil embolization of cerebral aneurysm. J Korean Neurosurg Soc 2017:60:518-26.

2 Ries T, Siemonsen S, Grzyska U, et al. Abciximab is a safe rescue therapy in thromboembolic events complicating cerebral aneurysm coil embolization. Stroke 2009;40:1750-7.

3 Dumont TM, Kan P, Snyder KV, et al. Adjunctive use of eptifibatide for complication management during elective neuroendovascular procedures. J Neurointerv Surg 2013;5:226-30.

4 Investigators TR. Effects of platelet glycoprotein IIb/IIla blockade with tirofiban on adverse cardiac events in patients with unstable angina or acute myocardial infarction undergoing coronary angioplasty. Circulation 1997;96:1445-53.

5 Bizzarri F, Scolletta S, Tucci E, et al. Perioperative use of tirofiban hydrochloride (Aggrastat) does not increase surgical bleeding after emergency or urgent coronary artery bypass grafting. J Thorac Cardiovasc Surg 2001;122:1181-5.

6 Kang H-S, Kwon BJ, Roh HG, et al. Intra-Arterial tirofiban infusion for thromboembolism during endovascular treatment of intracranial aneurysms. Neurosurgery 2008;63:230-8.

7 Dornbos D, Katz JS, Youssef $\mathrm{P}$, et al. Glycoprotein IIb/IIla inhibitors in prevention and rescue treatment of thromboembolic complications during endovascular embolization of intracranial aneurysms. Neurosurgery 2018;82:268-77.

8 Jeong HW, Jin S-C. Intra-Arterial infusion of a glycoprotein IIb/llla antagonist for the treatment of thromboembolism during coil embolization of intracranial aneurysm: a comparison of abciximab and tirofiban. AJNR Am J Neuroradiol 2013;34:1621-5.

9 Califf RM, Lincoff AM, Tcheng JE, et al. An overview of the results of the EPIC trial. Eur Heart J 1995;16 Suppl L:43-9.

10 Bangalore S, Toklu B, Kotwal A, et al. Anticoagulant therapy during primary percutaneous coronary intervention for acute myocardial infarction: a meta-analysis of randomized trials in the era of stents and P2Y12 inhibitors. BMJ 2014;349:g6419. 
Copyright 2021 BMJ Publishing Group. All rights reserved. For permission to reuse any of this content visit https://www.bmj.com/company/products-services/rights-and-licensing/permissions/

BMJ Case Report Fellows may re-use this article for personal use and teaching without any further permission.

Become a Fellow of BMJ Case Reports today and you can:

- Submit as many cases as you like

- Enjoy fast sympathetic peer review and rapid publication of accepted articles

- Access all the published articles

Re-use any of the published material for personal use and teaching without further permission

Customer Service

If you have any further queries about your subscription, please contact our customer services team on +44 (0) 2071111105 or via email at support@bmj.com.

Visit casereports.bmj.com for more articles like this and to become a Fellow 